\title{
Lattice model of reduced jamming by a barrier
}

\author{
Emilio N. M. Cirillo, ${ }^{1, *}$ Oleh Krehel, ${ }^{2, \dagger}$ Adrian Muntean, ${ }^{3, \ddagger}$ and Rutger van Santen ${ }^{4, \S}$ \\ ${ }^{1}$ Dipartimento di Scienze di Base e Applicate per l'Ingegneria, Sapienza Università di Roma, via A. Scarpa 16, I-00161, Roma, Italy \\ ${ }^{2}$ ICMS-Institute of Complex Molecular Systems, Department of Mathematics and Computer Science, Eindhoven University of Technology, \\ P.O. Box 513, 5600 MB Eindhoven, The Netherlands \\ ${ }^{3}$ Department of Mathematics and Computer Science, Karlstad University, Sweden \\ ${ }^{4}$ ICMS-Institute for Complex Molecular Systems, Faculty of Chemical Engineering, Eindhoven University of Technology, P.O. Box 513, \\ $5600 \mathrm{MB}$ Eindhoven, The Netherlands
}

(Received 18 February 2016; revised manuscript received 4 September 2016; published 14 October 2016)

\begin{abstract}
We study an asymmetric simple exclusion process in a strip in the presence of a solid impenetrable barrier. We focus on the effect of the barrier on the residence time of the particles, namely, the typical time needed by the particles to cross the whole strip. We explore the conditions for reduced jamming when varying the environment (different drifts, reservoir densities, horizontal diffusion walks, etc.). In particular, we discover an interesting nonmonotonic behavior of the residence time as a function of the barrier length. Besides recovering by means of both the lattice dynamics and the mean-field model well-known aspects like the faster-is-slower effect and the intermittence of the flow, we propose also a birth-and-death process and a reduced one-dimensional (1D) model with variable barrier permeability to capture the behavior of the residence time with respect to the parameters.
\end{abstract}

DOI: 10.1103/PhysRevE.94.042115

\section{INTRODUCTION}

Lattice models of particle flow may show surprisingly rich behavior even when only exclusion of a particle on the same site is considered [1]. Complex percolation behavior arises in particular at increased particle concentration (see Ref. [2] for a modern account on percolation theory, Ref. [3] for a case study related to the motion of colloids in narrow channels, and Ref. [4] for percolation effects in transportation in more general complex systems). In this paper, we introduce a twodimensional (2D) asymmetric simple exclusion random-walk model with diffusion and drift. The model aims at capturing the effect of the barrier positioned in the strip on the corresponding residence times, i.e., the time needed by a particle to cross the strip.

More precisely, we consider a vertical strip and measure the time that a particle entering the strip at the top side takes to exit the strip through the bottom side, under the assumption that the three other boundaries act as reflecting boundaries. This typical time will be called residence time.

We find an interesting nonlinear dependence on the length of this barrier when simulating the evolution of a high particle density in the strip. Instead of the expected increase in the residence time, at particular conditions we surprisingly notice a decrease in residence times with increasing barrier length. We mention that in the literature it has already been remarked that for nonequilibrium systems nonuniform dependence of current on a driving force may arise in the presence of a blockage $[5,6]$. Moreover, the effect we find reminds us of the Braess paradox, discovered when traffic flow unexpectedly decreases when an inhibitive traffic access barrier is removed (cf. Ref. [7]). This confirms once more the fact that as population densities and

\footnotetext{
*emilio.cirillo@uniroma1.it

†o.krehel@tue.nl

¥adrian.muntean@kau.se

${ }^{\S}$ R.A.v.Santen@tue.nl
}

the number of interactions among particles (agents, people, financial stocks, etc.) increase, so does the probability of emergent phenomena.

Our modeling approach and simulation results are potentially useful when trying to forecast the motion of pedestrian flows in open (heterogeneous) spaces. It has, for instance, been found that flocking of sheep [8,9] is helped by introducing a barrier before an exit point. Also, high-density particle flow through an orifice that leads to jamming has been found to have less jamming when a barrier is put in front of the orifice (see, for instance, Refs. [10] and [11] for crowd dynamics scenarios when the flow is improved by the presence of an obstacle in front of the exit). We have explored extensively in a previous paper (see Ref. [1]) the two-dimensional (2D) diffusion-drift strip lattice model used in this context, but without barriers. In this paper, our 2D lattice is perturbed by an immobile barrier with a fixed rectangular shape. At the top and of the bottom of the lattice, we assume the presence of reservoir (particle) densities. The size of the reservoirs controls the stochastic particle dynamics inside the lattice. Essentially, displacements can only occur toward unoccupied lattice sites. The displacement probabilities of the particles are possible in the four directions of the square lattice. The horizontal displacement probability perpendicular to the flow direction of the strip is $h / 2$, whereas $u$ and $d$ are the upward and downward displacement probabilities. The choices of $h, u$, $d$ are constrained by $h+u+d=1$.

The model describes the diffusion of particles in the lattice as well as a strongly nonlinear convection when drifts induced by $d-u \neq 0$ are introduced.

We focus on the case $d-u \geqslant 0$. When the drift (pointing out in the top-down direction) is nonzero, our stochastic simulations show a transition in the dependence of simulated average particle residence time as a function of the barrier length $W$. This transition is only found when the bottom reservoir density, say $\rho_{\mathrm{d}}$, exceeds a particular threshold value (see Fig. 8), while the range of barrier lengths of decreases in residence time depends on the choice of the drift value. In 
the absence of the drift, like transitions do not happen (as predicted, for instance, in Ref. [12] and references cited therein).

The analysis focuses on the relation between density profiles and residence time changes with the barrier width. It is the remarkable sudden change in density profile from convex to concave just behind the barrier that induces the residence time dependence to change from increasing to decreasing with increasing barrier width. The width of change relates to the value of the bottom boundary's prescribed density $\rho_{\mathrm{d}}$. It is worth mentioning two studies, see Refs. [13,14], of the behavior of 1D exclusion processes local inhomogeneities, in which density profiles similar to the ones we discuss in this paper are found.

Results are twofold: We will compare Monte Carlo simulation of the lattice model with numerical solutions of the 2D mean-field equations for the density profile and residence time. It appears that the lateral dependence of the density distribution is rather flat for $h$ that is sufficiently large [1] (in the simulation we shall use $h=0.5$ ). This we exploit by using a $1 \mathrm{D}$ differential mean-field equation to calculate residence time from laterally averaged simulated and calculated density distributions. This has the advantage that an analytical relation between residence time and density profile can be used to interpret the cause of the anomalous dependence of the residence time on the width of the barrier when the density $\rho_{\mathrm{d}}$ is larger than 0.5 and the drift is nonzero. When drift is zero for the 1D case an analytical solution to the residence time dependence can be found.

The paper is organized as follows. In Sec. II, we introduce the lattice model and the different methods to approach the barrier problem. We close this section with a short discussion of our main results. This is to be followed by the detailed presentation and analysis of our results in Secs. III A and III B. A summary of the main results and physics of the barrier introduction into the strip concludes the paper.

\section{MODELS AND METHODS}

In this section, we introduce the models we plan to study to address the problem discussed in the introduction and we also give a brief account of our main methods.

\section{A. Lattice dynamics}

The lattice model we discuss here is the same as the one introduced in Ref. [1], except for the presence of the barrier. We sketch, here, the definition of the mode and refer to Ref. [1] for details.

Take $L_{1}, L_{2}$ to be positive integer numbers and consider the $\operatorname{strip}\left\{1, \ldots, L_{1}\right\} \times\left\{1, \ldots, L_{2}\right\}$. We say that the coordinate directions 1 and 2 of the strip are respectively the horizontal and the vertical directions. We accordingly use the words top, bottom, left, and right. On the strip we consider a simple exclusion random walk with horizontal hopping probability $h$, up direction hopping probability $u$, and bottom direction hopping probability $d$, with $h+u+d=1$. Particles are inserted through the top and the bottom boundaries at rates mimicking fixed boundary densities $\rho_{\mathrm{u}}$ and $\rho_{\mathrm{d}}$.

Denote our vertical strip by $\Omega$. The impenetrable barrier is modeled by a rectangular region of width $W$ and height
$\mathrm{O}_{2}$ which is constantly occupied by particles at rest. Hence, particles moving on the lattice must do back steps and/or lateral jumps this region.

This model will be studied via Monte Carlo simulations. We will evolve the process for a time (termalization time) sufficiently long until the system reaches its stable steady state. After that, we measure the $2 \mathrm{D}$ density profile by averaging the occupation number at each site of the lattice (see, for instance, Fig. 2).

Moreover, we shall also measure the residence time by averaging, at stationarity, the time needed by a particle entered through the top boundary to exit through the bottom one. In this computation, the top boundary condition will be chosen to be $\rho_{\mathrm{u}}=1$ so that particles will not be allowed to leave the system through the top boundary.

In the study of the residence time we shall find two very different behaviors corresponding to the case when the particle dynamics will be either biased or not along the vertical direction. A special role will be played by the parameter

$$
\delta=\frac{d-u}{d+u}
$$

which will be called drift.

For more details, we refer again the reader to Ref. [1] where a complete account of this approach is provided.

\section{B. Mean-field dynamics}

A mean-field approximation of the lattice model can be derived as in Ref. [1], using arguments very much inspired from Ref. [15]. The difference here is the presence of the barrier, which is treated as follows. We omit the details.

We denote our vertical strip by $\Omega$ and refer to the internal barrier as $\mathcal{O}$; see Fig. 1 for a sketch of the geometry. The density profile can be well approximated as a solution to the mean-field equation

$$
\frac{\partial \rho}{\partial t}=\frac{h}{2} \frac{\partial^{2} \rho}{\partial y^{2}}+\frac{1-h}{2} \frac{\partial^{2} \rho}{\partial x^{2}}-\delta(1-h) \frac{\partial}{\partial x}[\rho(1-\rho)]
$$

in $\Omega \backslash \mathcal{O}$, endowed with the initial condition

$$
\rho(0, y, x)=0 \text { in } \Omega \backslash \mathcal{O}
$$

and the boundary conditions

$$
\rho(t, y, 0)=\rho_{\mathrm{u}}, \rho\left(t, y, L_{2}\right)=\rho_{\mathrm{d}},
$$

and

$$
\frac{\partial \rho(t, 0, x)}{\partial y}=\frac{\partial \rho\left(t, L_{1}, x\right)}{\partial y}=\nabla \rho n_{\partial \mathcal{O}}=0 .
$$

Here $n_{\partial \mathcal{O}}$ denotes the outer normal along the boundary of the barrier $\mathcal{O}$.

This mean-field model, a nonlinear diffusion-drift equation, is approximated via a finite element approach. The problem (2.2)-(2.5) is integrated numerically and the density profile $\rho(y, x)$ is found. Then the residence time is computed by means of Eq. (2.6). We used the finite-element numerics toolbox DUNE [16] to implement a solver for the model. We used quadratic Lagrange elements and the Newton method to deal with the nonlinear drift term. 


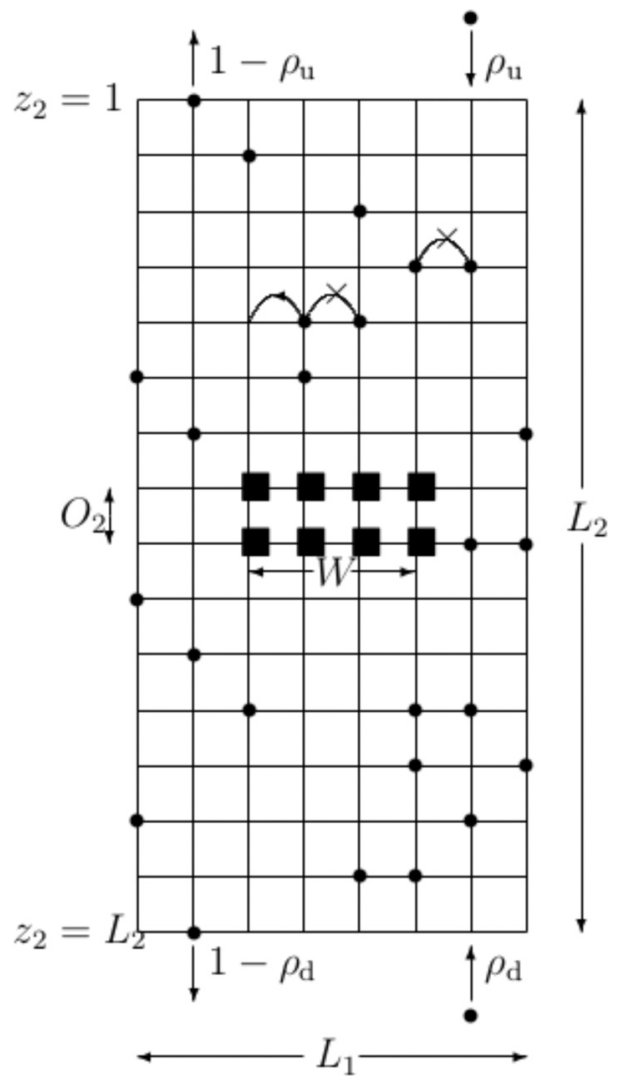

FIG. 1. Schematic representation of the model in the presence of the barrier. Solid squares represent the particles at rest modeling the barrier.

Simulations indicate that there may not be too much dependence in the density profile on the $y$ variable and we can approximate the $2 \mathrm{D}$ density profile with its $1 \mathrm{D}$ counterpart $\rho(x)$ that we obtain by integrating out the $y$ variable. We shall misuse the notation and denote by $\rho$ both the 2D density distribution and the 1D density profile. This 1D density profile can be then used to calculate the residence time estimate that is given from the mean-field expression

$$
R=-\frac{2}{(1-h) \partial_{x} \rho(0)} \int_{0}^{L_{2}} \rho(x) d x .
$$

This expression [1, Eq. (5.35)] shows that the average particle residence time is determined by the derivative of the density at the entrance of the strip and the integrated density. The convex to concave density profile change behind the barrier in Fig. 2 indicates a large change in the particle density, that, as we will see, is responsible to a significant extent to the transition behavior of the residence time. We have shown in Ref. [1] that the mean-field equation (2.2) is only valid in a limited regime of the parameter space, where a birth-anddeath random-walk model providing an alternative approach to calculate the residence time is proposed.

\section{One-dimensional reduction}

We propose a twofold reduction of the mean-field model. This way, we reduce the dimensionality of the model from 2D to $1 \mathrm{D}$ and compensate, based on an effective transport coefficient, for the presence of the obstacle. For this we use a porous media modeling approach where parameters like obstacle porosity and tortuosity will be used in the 1D context. Similar arguments are indicated, for instance, in Ref. [17].

It occurs to us that there may be not too much dependence in the density profile on the $y$ variable and we can approximate the $2 \mathrm{D}$ density profile with its $1 \mathrm{D}$ counterpart that we obtain by integrating out the $y$ variable. After integration, the $x$ coordinates that correspond to the place where the barrier was in two dimensions are designated to have a smaller diffusion coefficient to account for that obstacle.

In our initial approximation, we consider the diffusion coefficient and the drift to be porosity and tortuosity based via the coefficient

$$
\lambda(x)= \begin{cases}F(h) \frac{L_{1}-W}{L_{1}} & x \in\left[\frac{L_{2}-O_{2}}{2}, \frac{L_{2}+O_{2}}{2}\right], \\ 1 & \text { otherwise. }\end{cases}
$$

For convenience we also let $\alpha:=F(h)\left(L_{1}-W\right) / L_{1}$. Here, the ratio $\left(L_{1}-W\right) / L_{1}$ is the porosity, while $F(h)$ is the currently unknown function of the horizontal displacement probability $h$. This plays the role of the tortuosity. It is expected that $F(h) \in(0,1)$. In this very basic approximation porosity and tortuosity effects are independent (multiplicative), so that the no barrier case is recovered for $W=0$ and $F(h)=1$ in the expression (2.7). An increase in $W$ results in a decrease in $\lambda(x)$ in the region $x \in\left[\left(L_{2}-O_{2}\right) / 2,\left(L_{2}+O_{2}\right) / 2\right]$, which is also the expected behavior from the lattice model.

The 1D mean-field equation reads

$$
\frac{d}{d x}\left[\lambda(x)\left(\frac{1}{2} \frac{d \rho}{d x}-\delta \rho(1-\rho)\right)\right]=0
$$

with the boundary conditions

$$
\rho(0)=1 \text { and } \rho\left(L_{2}\right)=\rho_{\mathrm{d}} .
$$

On the basis of the density profile obtained by solving (2.8), it is possible to compute the residence time via a standard argument; see, e.g., Ref. [1], Sec. 5.6. We find

$$
R=-\frac{2}{\rho^{\prime}(0)} \int_{0}^{L_{2}} \rho(x) d x,
$$

which is analogous to Eq. (2.6).

We will see in the next section that the reduced model in (2.8) and (2.9) is a convenient approximation of the $2 \mathrm{D}$ mean-field model with barrier in the zero drift case. In this context the model will be solved explicitly and the density profile will be computed. Then we will compute the residence time using again (2.6).

\section{RESULTS}

In this section we will present our results discussing the zero drift case and nonzero drift case separately.

For the zero drift case the analytical solution of the 1D barrier problem (2.8) gives the density profile. In Secs. III A 1 and III A 2 we compare this solution to the horizontally averaged 2D density distributions computed for the lattice model and for the mean-field model and a perfect match is found. In Sec. III A 3 the associated residence time is computed via Eq. (2.10) and via the birth-and-death model [1]. These 
(a)

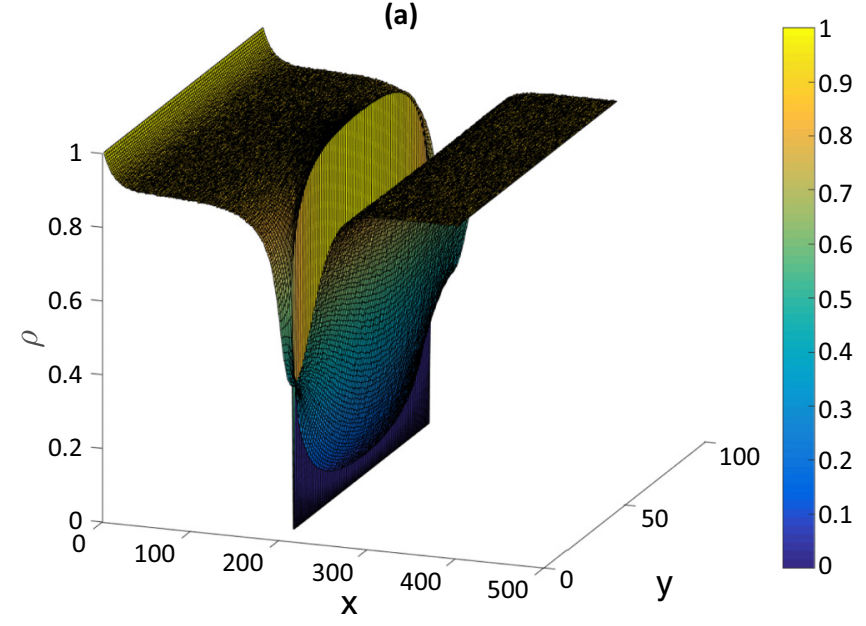

(c)

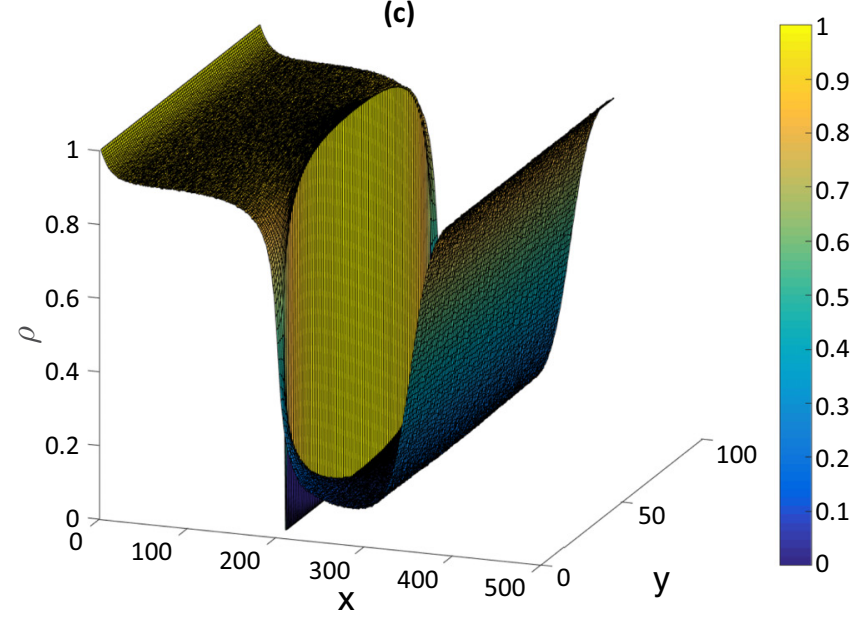

(e)

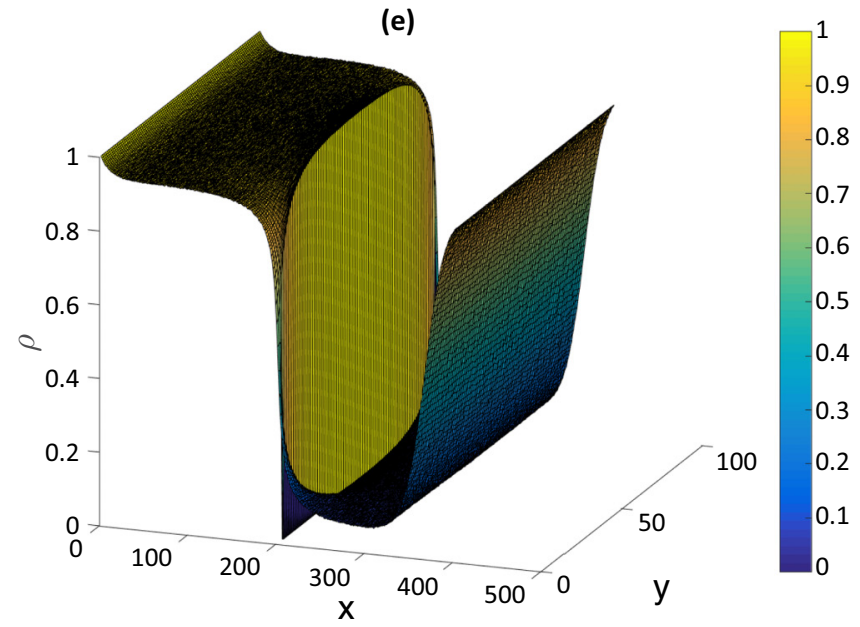

(b)

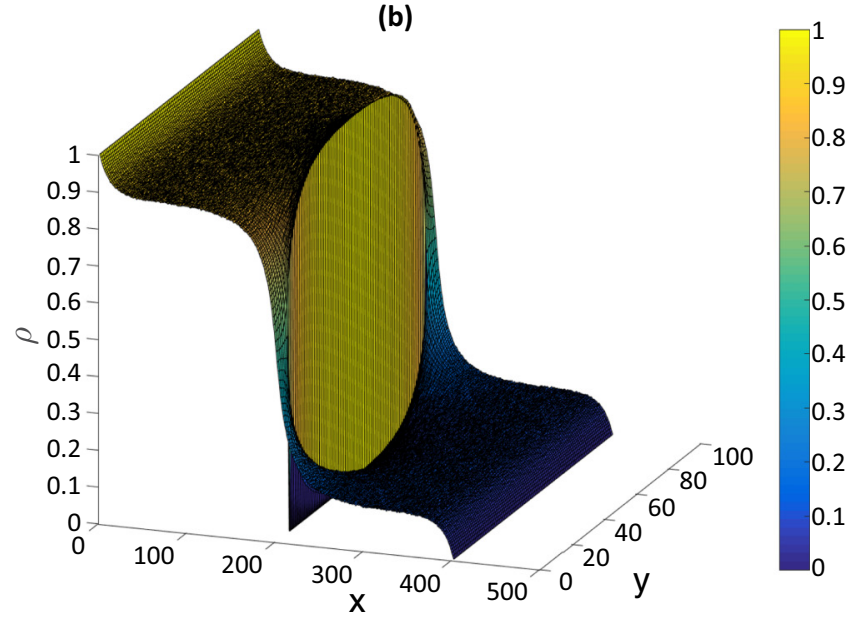

(d)

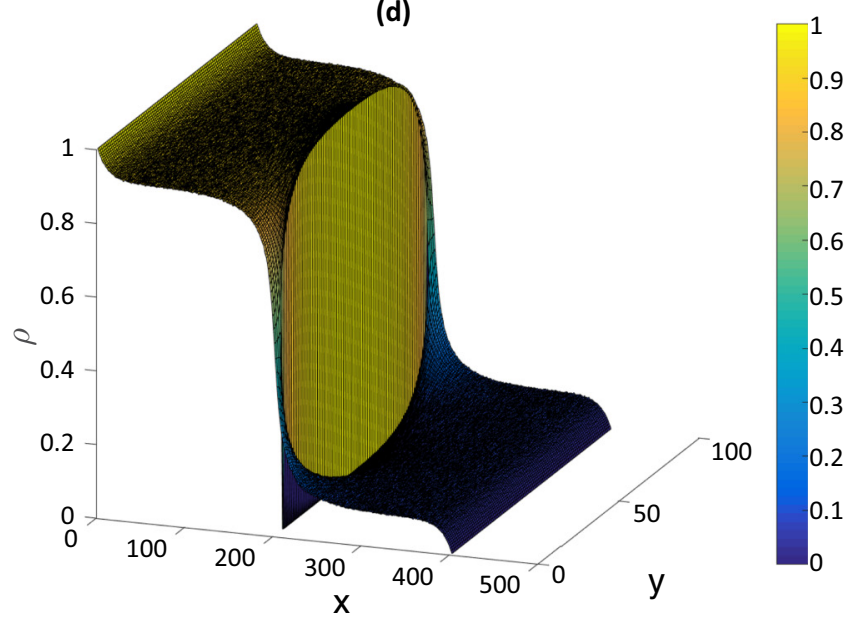

(f)

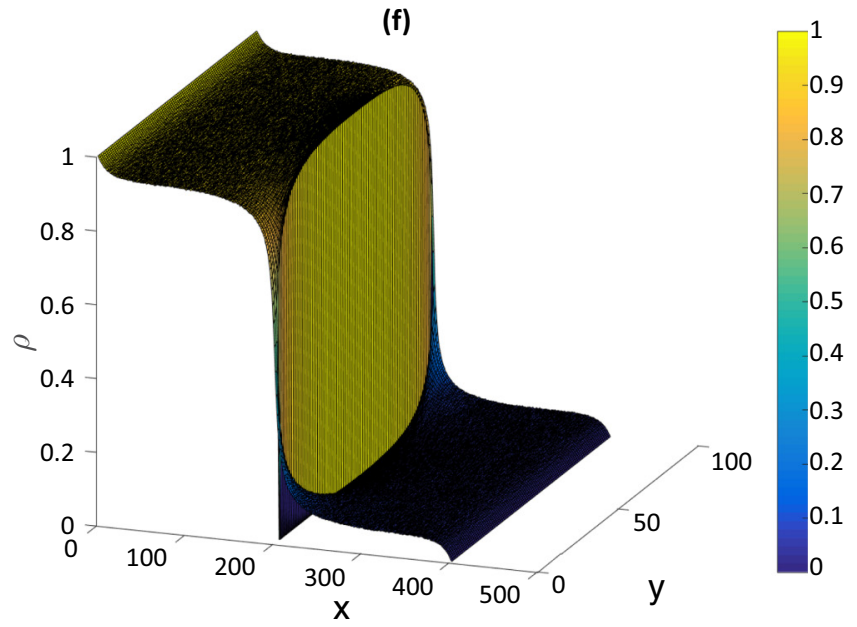

FIG. 2. Simulated 2D density profiles for the lattice model in the presence of the barrier: comparison between low and high bottom boundary densities. On the left $\rho_{\mathrm{d}}=0.9$ and on the right $\rho_{\mathrm{d}}=0.0$, three values of the barrier width are compared: $W=85$ (top), $W=90$ (middle), and $W=95$ (bottom). The corresponding simulated mean residence time is equal to 81359.8 (b), 101390 (d), 146403 (f) for $\rho_{\mathrm{d}}=0.0$; and 146678 (a), 119865 (c), and 162350 (e) for $\rho_{\mathrm{d}}=0.9$. The other model parameters used in the simulation are $L_{1}=100, L_{2}=400, h=0.5, \delta=0.05$, $\rho_{\mathrm{u}}=1, O_{2}=3$. 
results will be compared to the averaged $2 \mathrm{D}$ density profile obtained via the Monte Carlo simulation and the associated residence time.

In Sec. III B, for the nonzero drift case, for which no analytical 1D model could be developed, the 2D Monte Carlo stationary density distribution is reduced to a $1 \mathrm{D}$ profile via lateral averaging. Moreover, such a profile is used to estimate the residence time via mean-field and birth-and-death methods. Results are compared to the Monte Carlo estimates for the residence time. As for the nonzero drift case, a short interpretation of the data is given.

In this case, we find that the residence time depends not monotonically on the barrier width provided the bottom reservoir density is large enough; more precisely, when $\rho_{\mathrm{d}}$ is larger than 0.5 , the residence time behavior changes from the monotonic increase with increase of $W$ to a nonmonotonic behavior: It decreases until a critical value $W_{\mathrm{c}}$ is reached and increases beyond it. In the absence of the drift, alike transitions do not happen (as predicted, for instance, in Ref. [12] and references cited therein). It is worth noting that in the absence of drift, the dependence on the barrier width always turns into a monotonic increase of the residence time with increasing width.

\section{A. Zero drift case}

We consider the lattice model introduced in Sec. II A on the lattice strip of size $L_{1} \times L_{2}$ in absence of drift, namely, for $\delta=0$. Our simulations will be run mainly for $L_{1}=100$, $L_{2}=400, h=0.5, \rho_{\mathrm{u}}=1$, and $\rho_{\mathrm{d}}=0,0.9$. But in some cases we shall also consider the values $L_{1}=200$ and $h=0.3,0.4$. Our barrier is of size $\mathrm{W} \times \mathrm{O}_{2}$ and is placed in the middle of the strip. The typical values used in the simulations for the width $W$ of the barrier are $10,20, \ldots, 90$. Its height $O_{2}$ will always be equal to 3 .

\section{Solution to the $1 D$ model}

For $\delta=0$ the model in Sec. II C simplifies and a thorough analytical treatment is possible. The 1D equation (2.8) is a linear diffusion equation with a piecewise constant diffusion coefficient. Its solution is piecewise linear on intervals $\left[0,\left(L_{2}-\right.\right.$ $\left.\left.O_{2}\right) / 2\right],\left[\left(L_{2}-O_{2}\right) / 2,\left(L_{2}+O_{2}\right) / 2\right],\left[\left(L_{2}+O_{2}\right) / 2, L_{2}\right]$, and we can express it in the form

$$
\rho(x)=\rho_{\mathrm{u}} T_{0}(x)+a T_{1}(x)+b T_{2}(x)+\rho_{\mathrm{d}} T_{3}(x),
$$

where the coefficients $a$ and $b$ are the unknowns. The functions $T_{i}$ are the linear pyramid functions. Their derivatives are $T_{0}^{\prime}(x)=-2 /\left(L_{2}-O_{2}\right)$ on $\left[0,\left(L_{2}-O_{2}\right) / 2\right]$ and 0 otherwise,

$$
\begin{gathered}
T_{1}^{\prime}(x)= \begin{cases}\frac{2}{L_{2}-O_{2}} & \text { on }\left[0,\left(L_{2}-O_{2}\right) / 2\right], \\
\frac{-1}{O_{2}} & \text { on }\left[\left(L_{2}-O_{2}\right) / 2,\left(L_{2}+O_{2}\right) / 2\right], \\
0 & \text { otherwise }\end{cases} \\
T_{2}^{\prime}(x)= \begin{cases}\frac{1}{O_{2}} & \text { on }\left[\left(L_{2}-O_{2}\right) / 2,\left(L_{2}+O_{2}\right) / 2\right], \\
-\frac{2}{L_{2}-O_{2}} & \text { on }\left[\left(L_{2}+O_{2}\right) / 2, L_{2}\right], \\
0 & \text { otherwise. }\end{cases}
\end{gathered}
$$

and $T_{3}^{\prime}(x)=2 /\left(L_{2}-O_{2}\right)$ on $\left[\left(L_{2}+O_{2}\right) / 2, L_{2}\right]$ and 0 otherwise. After substituting (3.11) into (2.8), multiply both sides by $T_{1}(x)$ and $T_{2}(x)$ and then integrate. This yields the following equations:

$$
\int_{0}^{L_{2}} \rho^{\prime}(x) D(x) T_{1}^{\prime}(x) d x=0 \text { and } \int_{0}^{L_{2}} \rho^{\prime}(x) D(x) T_{2}^{\prime}(x) d x=0 .
$$

From here it follows that

$$
\int_{0}^{\frac{L_{2}-O_{2}}{2}}\left(\rho_{\mathrm{u}} T_{0}^{\prime}+a T_{1}^{\prime}\right) T_{1}^{\prime} d x+\int_{\frac{L_{2}-O_{2}}{2}}^{\frac{L_{2}+O_{2}}{2}}\left(a T_{1}^{\prime}+b T_{2}^{\prime}\right) \alpha T_{1}^{\prime} d x=0
$$

and

$$
\int_{\frac{L_{2}-O_{2}}{2}}^{\frac{L_{2}+O_{2}}{2}}\left(a T_{1}^{\prime}+b T_{2}^{\prime}\right) \alpha T_{2}^{\prime} d x+\int_{\frac{L_{2}+O_{2}}{2}}^{L_{2}}\left(b T_{2}^{\prime}+\rho_{\mathrm{d}} T_{3}^{\prime}\right) T_{2} d x=0 .
$$

After integration, we obtain

$$
a=\frac{\rho_{\mathrm{u}}+\rho_{\mathrm{d}}+\rho_{\mathrm{u}} \beta}{2+\beta} \text { and } b=\frac{\rho_{\mathrm{u}}+\rho_{\mathrm{d}}+\rho_{\mathrm{d}} \beta}{2+\beta}
$$

with

$$
\beta=\frac{O_{2}}{\alpha\left(L_{2}-O_{2}\right)} .
$$

We remark that the deviations in the density profile from the straight line are symmetric. (See, e.g., Fig. 4 for an example of a simulation.) Indeed, by summing the coefficients in (3.12) we obtain $a+b=\rho_{\mathrm{u}}+\rho_{\mathrm{d}}$ and, hence,

$$
a-\frac{\rho_{\mathrm{u}}+\rho_{\mathrm{d}}}{2}=\frac{\rho_{\mathrm{u}}+\rho_{\mathrm{d}}}{2}-b=\frac{\left(\rho_{\mathrm{u}}-\rho_{\mathrm{d}}\right) \beta}{4+2 \beta} .
$$

Having obtained the analytical expression for $\rho(x)$, we can compute the 1D mean-field residence time approximation by using (2.10). Indeed, some simple algebra yields

$$
R=\frac{\left(\rho_{\mathrm{u}}+\rho_{\mathrm{d}}\right) L_{2}\left(L_{2}-O_{2}\right)\left[2+\frac{2 O_{2}}{\alpha\left(L_{2}-O_{2}\right)}\right]}{\rho_{\mathrm{u}}-\rho_{\mathrm{d}}},
$$

where, we recall, $\alpha=F(h)\left(L_{1}-W\right) / L_{1}$. In the case $\alpha=1$, i.e., no barrier, the expression of the residence time simplifies to

$$
R=\frac{\left(\rho_{\mathrm{u}}+\rho_{\mathrm{d}}\right) 2 L_{2}^{2}}{\rho_{\mathrm{u}}-\rho_{\mathrm{d}}},
$$

which is an agreement with Eq. (5.39) in Ref. [1].

We note the following: According to (3.14), the residence time increases with increasing value of $\rho_{\mathrm{d}}$. Additionally, the effect of $W$ on the residence time disappears when $L_{2}$ goes to infinity. Moreover, from (3.14), the residence time uniformly increases as $W$ increases. Note also that the $W$ dependence can be also seen purely as $W / L_{1}$. This is a limitation of our simple approximation to the diffusion coefficient, since in our simulations we see an effect of different values of $W$ on the residence time, even with the same $W / L_{1}$ ratio (see Sec. III A 3).

\section{Density profile}

Now, we discuss how the density profile obtained from (3.11) compares to the one obtained by averaging the 2D Monte Carlo simulation. The results are shown in Fig. 3 in the case $W=70$. The parameters we used in the computation are listed in the caption.

The match between the Monte Carlo and the analytical result is perfect. For the $1 \mathrm{D}$ model we had to optimize 


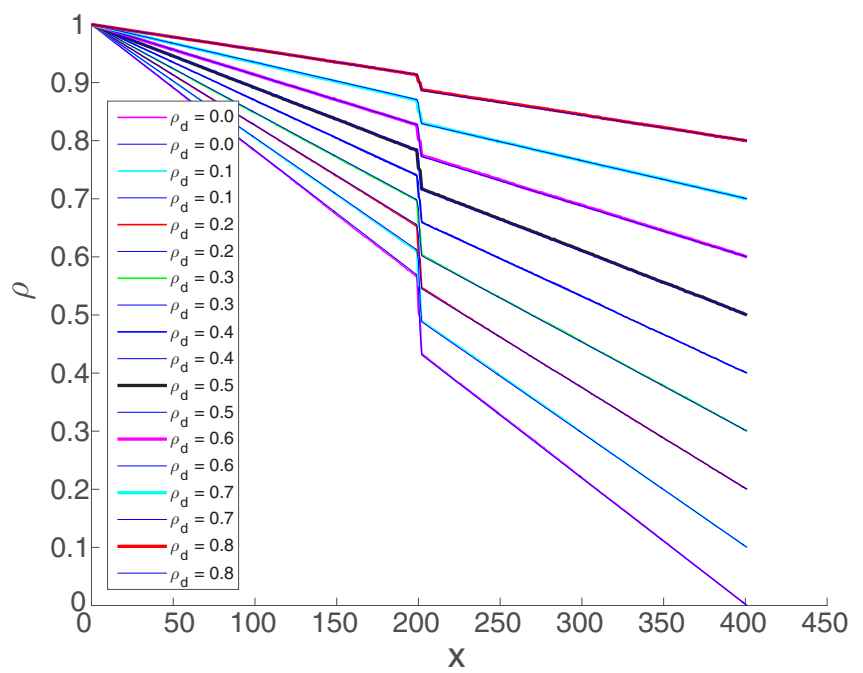

FIG. 3. Comparison between the density profile obtained by averaging the $2 \mathrm{D}$ lattice simulation and the analytical solution of the 1D mean-field equation. Parameters: $L_{1}=100, L_{2}=400, h=0.5$, $\delta=0, W=70, O_{2}=3, \rho_{\mathrm{u}}=1$, and $\rho_{\mathrm{d}}$ as listed in the inset. For the $1 \mathrm{D}$ model the tortuosity coefficient has been chosen equal to 0.45 for all the values of $\rho_{\mathrm{d}}$. Thick lines correspond to Monte Carlo data for the lattice model and thin lines correspond to the analytical solution of the 1D model.

on the tortuosity coefficient by choosing $F=0.45$ for this comparison, but we stress that the same value has been used for all the choices of the bottom boundary density plotted in the picture. Although this value resulted in a good match, the question of the explicit dependence $F(h)$ still remains open.

The size of the jump in the averaged density profile, which can be observed in the figure, obviously depends on the width of the barrier. This dependence is analyzed in Fig. 4, where we plot the averaged Monte Carlo density profile for the 2D lattice model for different values of $W$. The two plots show our results for $\rho_{\mathrm{d}}=0$ (top panel) and $\rho_{\mathrm{d}}=0.9$ (bottom panel). It is worth remarking that, as we expected, in both cases the size of the jump increases with the barrier width. But we stress that the qualitative behavior of the graph does not change with $\rho_{\mathrm{d}}$. This fact is particularly relevant and it is key in our explanation for the different behaviors that we shall find in the biased (nonzero drift) case.

\section{Residence time}

The above discussion shows that the 2D stationary density profile can be found by averaging the Monte Carlo data for the 2D lattice model or by solving the mean-field model (2.2). Moreover, by averaging along the horizontal axis this 2D profile, we find a $1 \mathrm{D}$ profile which can be perfectly fitted with the $1 \mathrm{D}$ model proposed in Sec. II C by choosing the correct tortuosity coefficient. Such a 1D density profile can be used as an input to estimate the residence time.

This estimate can be achieved via the mean-field approximation provided in (2.6). But we shall also use a different approach proposed in Ref. [1] and based on a birth-and-death model. The main idea is that of predicting the residence time via a $1 \mathrm{D}$ model in which a particle performs a simple random
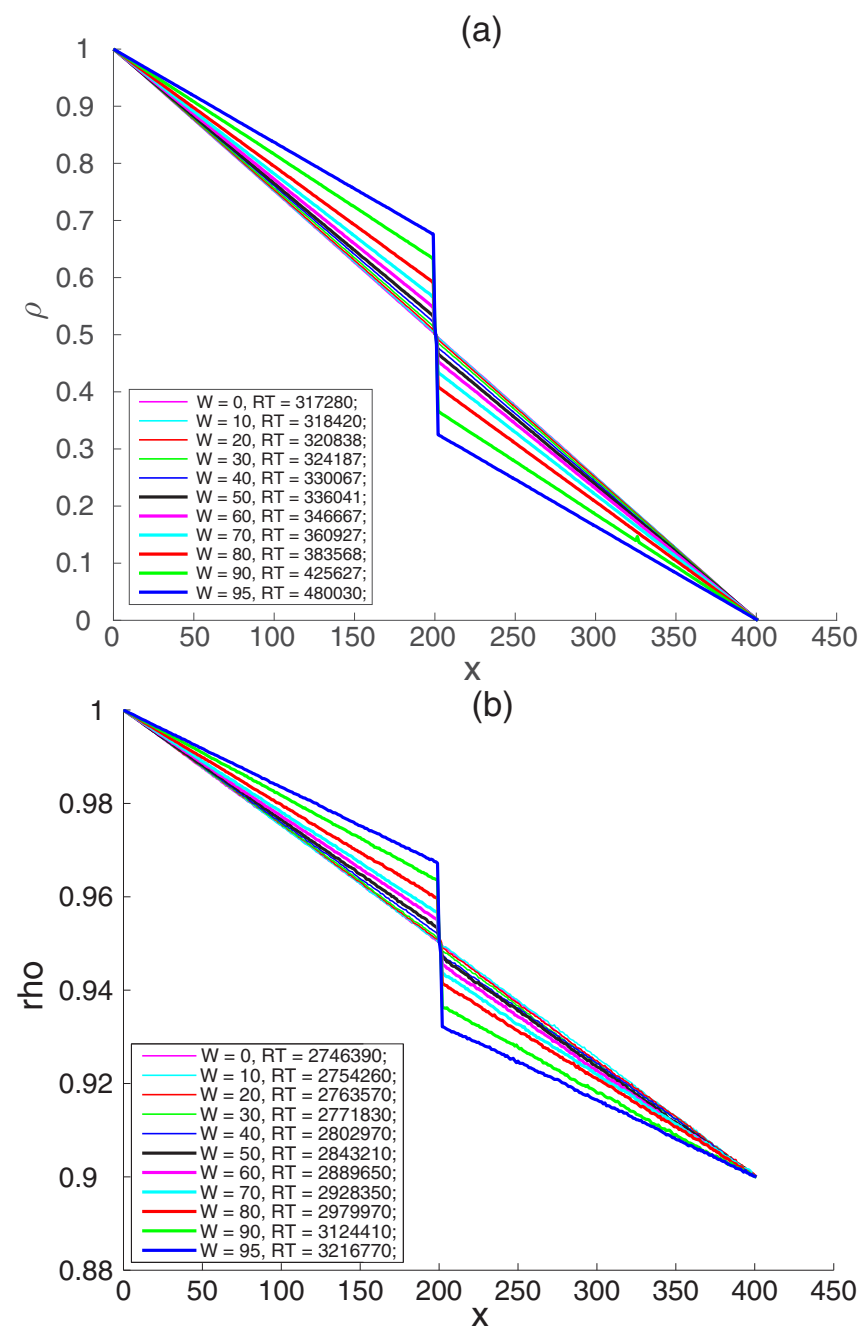

FIG. 4. Density profile obtained by averaging the $2 \mathrm{D}$ lattice simulation: comparison for different values of $W$. Parameters: $L_{1}=$ $100, L_{2}=400, h=0.5, \delta=0, \rho_{\mathrm{u}}=1, \rho_{\mathrm{d}}=0$ (a) and $\rho_{\mathrm{d}}=0.9$ (b), $\mathrm{O}_{2}=3$, and $W$ as listed in the inset. In the inset we have also listed the Monte Carlo-averaged residence time data discussed in Sec. III A 3.

walk in the vertical direction with jumping probabilities defined in terms of the stationary density profile measured for the 2D lattice model. In particular it has been deduced the prediction [Ref. [1], Eq. (4.20)] for the residence time based on the birth-and-death model defined in Ref. [1], Eq. (5.28). In that paper, due to the absence of a barrier, the reduction to $1 \mathrm{D}$ is rather obvious, since the density profile does not depend on the horizontal coordinate. As in the case of the mean-field approximation (2.6), we shall also use this birth-and-death approach here starting from the horizontally averaged density introduced above Eq. (2.6).

In Figs. 5 and 6, we compare the Monte Carlo measurement of the residence time (LA) to the birth-and-death (BD) and mean-field (MF) estimates based on the horizontally averaged 1D density profile $\rho$ of the 2D simulation of a flow through a strip with an obstacle in the middle. On the horizontal axis we have the barrier width and on the vertical axis the mean residence time. The formulas for the both residence time estimates can be found in Ref. [1]; more precisely, see 


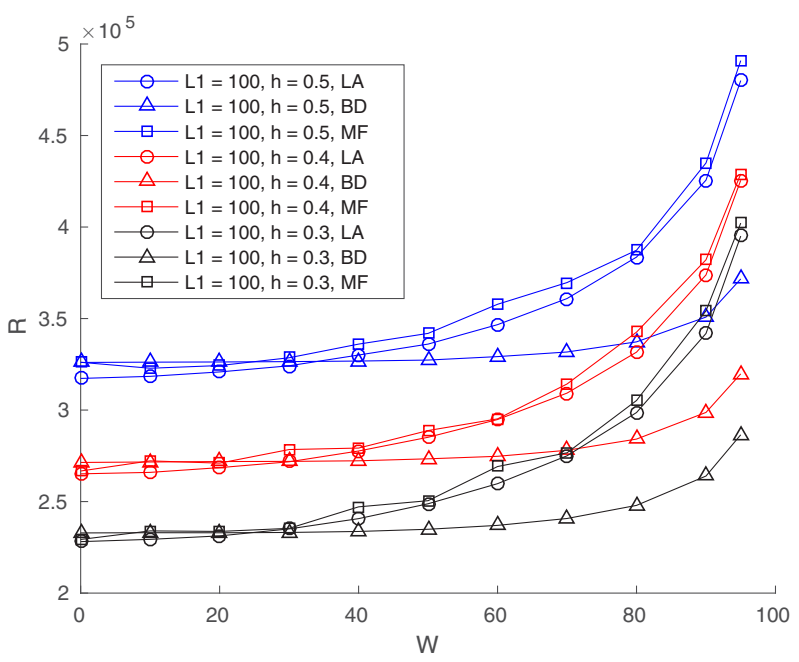

FIG. 5. The birth-and-death and mean-field approximations to the actual measured mean residence time (labeled LA). Parameters: $L_{1}=100, L_{2}=400, \delta=0, \rho_{\mathrm{u}}=1, \rho_{\mathrm{d}}=0, O_{2}=3$, and $h=0.5$.

Eqs. (5.32) and (5.39) in Ref. [1] respectively for the BD and the MF approximations.

As we can see, the quality of the approximation is influenced heavily by the value of $\rho_{\mathrm{d}}$. For $\rho_{\mathrm{d}}=0$, the MF approximation works very well, while the $\mathrm{BD}$ approximation gets worse when the width of the barrier is increased. For $\rho_{\mathrm{d}}=0.9$, the MF approximation overestimates by a lot, while the $\mathrm{BD}$ approximation is a bit better, but still not very precise. This result is consistent with what it has been found in Ref. [1] in the absence of a barrier: In the absence of drift, provided $h$ is large enough (here we are using $h=0.5$ ), the BD prediction is better than the MF one in those situations in which clogging is present. There, in the absence of obstacles, clogging was introduced by increasing the value of the bottom boundary density.

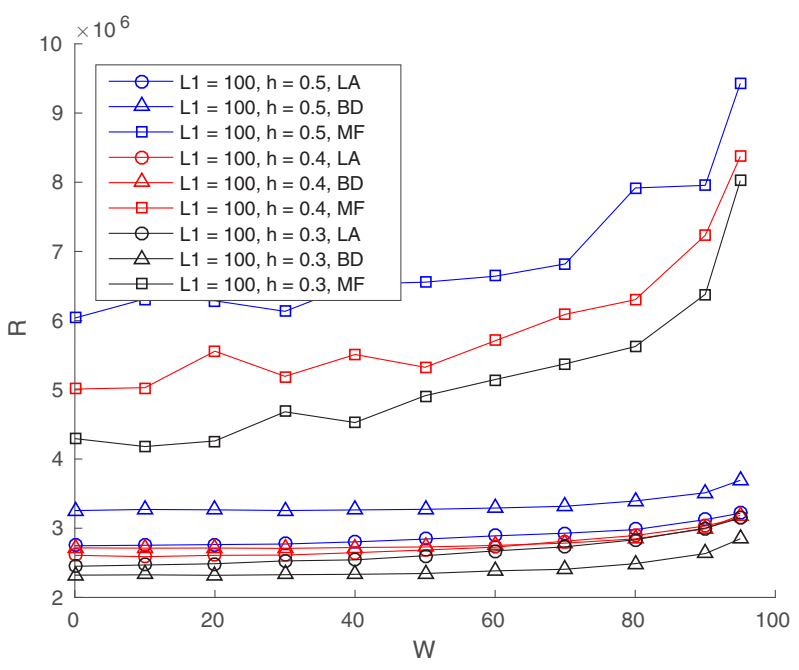

FIG. 6. The birth-and-death and mean-field approximations to the actual measured mean residence time (labeled LA). Parameters: $L_{1}=100, L_{2}=400, \delta=0, \rho_{\mathrm{u}}=1, \rho_{\mathrm{d}}=0.9, O_{2}=3$, and $h=0.5$.
From this it follows that we cannot expect to get precise residence time estimates based on the analytical solution of our 1D model for the case of zero drift. But we can still hope to reproduce the density profiles well.

As a final remark, on which we shall come back in the discussion in Sec. IV in connection with the results we will find in the nonzero drift situation, we note that the behavior of the residence time with the barrier width is absolutely trivial. Indeed, it stays more or less constant until half the horizontal width is reached, and then it increases sharply.

\section{B. Nonzero drift case}

In the zero drift case, residence time and current are determined by diffusion and hence by the gradient in particle density. For this case in the previous subsection we have shown that the barrier reduces density gradients in the strip. This reduced-density gradient compared to the no-barrier case is the reason for the longer residence time when a barrier is present.

The physically very different responses for the case of nonzero drift upon the presence of a barrier relates to the very different particle density profile in the strip when drift dominates versus diffusion. Since the stationary density distribution, even in presence of a barrier, will poorly depend on the horizontal spatial coordinate, we will often use 1D arguments based on the $1 \mathrm{D}$ density profile obtained by averaging the $2 \mathrm{D}$ density distribution on the horizontal spatial coordinate.

As is well known, for diffusion the particle density profile decreases linearly between its high value at entrance of the strip and its low value at the exit. This can be observed, for the no-barrier case, in Fig. 4. When drift is finite, the density profile becomes very different (e.g., see Fig. 10).

In $1 \mathrm{D}$, when no barrier is present, the particle density profile in the strip is determined by the condition that current is maximum [12]. The drift contribution to the current is proportional to $\rho(1-\rho)$; see (2.8) and Ref. [1], Eq. (5.34), which is maximum at $\rho=0.5$. Therefore, when possible, the particle density in the strip will tend to be flat and equal to 0.5 .

In our case the inlet density of the strip $\rho_{\mathrm{u}}=1$ is kept constant, but the outlet density $\rho_{\mathrm{d}}$ is varied. One has to distinguish the cases $\rho_{\mathrm{d}}$ less than or equal to 0.5 and $\rho_{\mathrm{d}}>$ $0.5[1,12,18]$. When $\rho_{\mathrm{d}}$ is less than 0.5 and the drift dominates, except for a small region close to the boundaries, the density profile is nearly constant and close to 0.5 . The density in the flat part of the profile will not change unless $\rho_{\mathrm{d}}$ exceeds 0.5 . Then the value of the flat density part in the strip will increase to $\rho_{\mathrm{d}}$ (see the case without barrier in Fig. 10). This can be considered the onset region of percolation, since when $\rho_{\mathrm{d}}>0.5$ the $\rho(1-\rho)$ term decreases the current decreases and the residence time increases.

The different responses of residence time on barrier length for the zero and nonzero drift cases relate to the very different physics that determines current in the two cases: density gradient when diffusion dominates and $\rho(1-\rho)$ term when drift dominates. In the next section simulations of the dependence of the residence time on barrier width will be presented. We will discuss under which conditions the residence time will behave nonlinearly and will show a minimum as a function of barrier width. In the following subsection we will relate this 


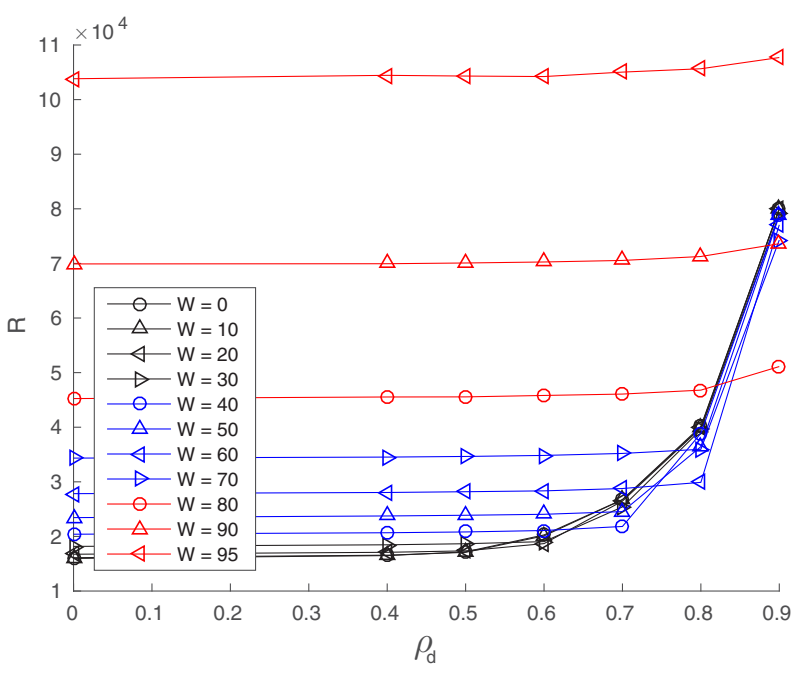

FIG. 7. Simulated mean residence time for the 2D lattice model as a function of $\rho_{\mathrm{d}}$ for the different values of $W$ listed in the inset. Parameters: $L_{1}=100, L_{2}=400, h=0.5, \delta=0.1, \rho_{\mathrm{u}}=1$, and $\mathrm{O}_{2}=3$.

nonlinear residence time behavior to changes in the density profiles. After a discussion of the changes in density profiles when barrier width changes we will analyze these changes using the $1 \mathrm{D}$ model.

\section{Residence time}

We consider the lattice model introduced in Sec. II A on the lattice strip $L_{1} \times L_{2}$ in the presence of drift, namely, for $\delta>0$. Our simulations will be run mainly for the same parameters as those used in Sec. III A. Details will be given in the figure captions.

The dependence of the residence time on the barrier width is shown in Figs. 7 and 8. Again, it is essential to consider the cases where $\rho_{\mathrm{d}}$ is smaller or larger than 0.5 .

Figure 7 shows simulated residence time as a function of $\rho_{\mathrm{d}}$. In the inset the values of $W$ are shown. When there is no barrier, $W=0$, the residence time increases only once $\rho_{\mathrm{d}}$ exceeds 0.5 . As discussed above this is due to the onset of percolation. As long as $\rho_{\mathrm{d}}$ does not exceed a critical value, when $W$ is different from zero the residence time increases with increasing value of $W$. As for the case $W=0$, the residence time remains independent of $\rho_{\mathrm{d}}$ until $\rho_{\mathrm{d}}$ exceeds this particular critical value. This critical value of $\rho_{\mathrm{d}}$ increases as $W$ becomes larger.

Interestingly at this critical value of the outlet boundary density $\rho_{\mathrm{d}}$ the residence time in the presence of a barrier, as long as $W$ does not exceed a critical value $W_{\mathrm{c}}$, is lower than the residence time for the no-barrier strip $(W=0)$.

In the case where $\rho_{\mathrm{d}}$ is kept constant, the residence time will always increase with $W$ as long as $\rho_{\mathrm{d}}$ is less than 0.5 . However, if $\rho_{\mathrm{d}}$ is larger than 0.5 , there is a critical value $W_{\mathrm{c}}$ of the barrier width below which the residence time will decrease. The value of $W_{\mathrm{c}}$ increases with $\rho_{\mathrm{d}}$. This defines a region of $\rho_{\mathrm{d}}-W$ values where the residence time will decrease below that of the no-barrier case. This is illustrated diagrammatically in Fig. 8.

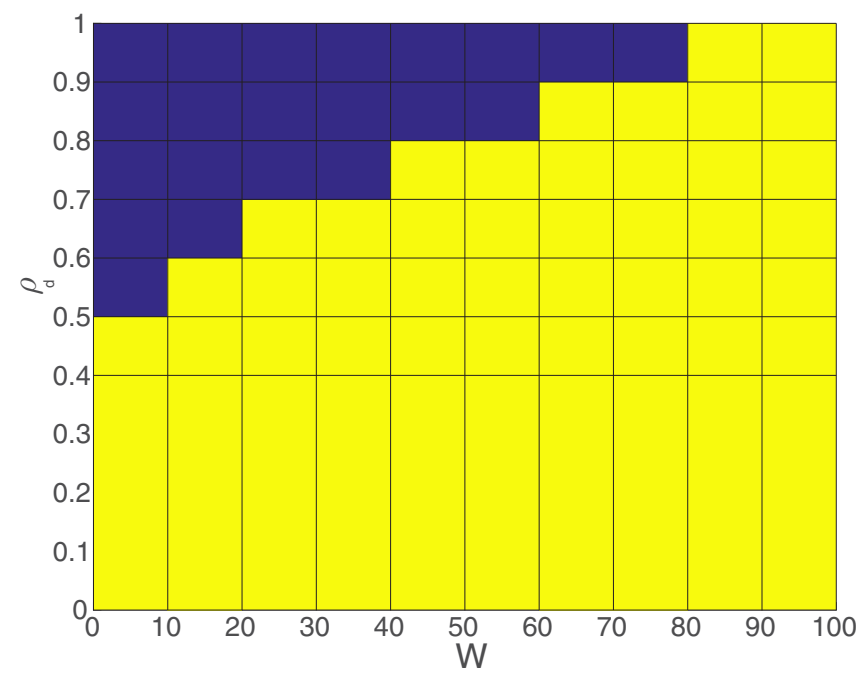

FIG. 8. Diagram of the residence time behavior with respect to the bottom boundary density $\rho_{\mathrm{d}}$ and the barrier width $W$. Here we plot the sign of the numerical derivative of $R$ with regard to $W$. Yellow part: the residence time increases with increase in $W$. Blue part: the residence time decreases with increase in $W$. Other parameters: $L_{1}=400, L_{2}=100, \delta=0.1, h=0.5$.

The critical value $W_{\mathrm{c}}$, below which the residence time has a minimum, is also a function of the drift. Figure 9 illustrates the change in residence time as a function of $W$ for the case $\rho_{\mathrm{d}}=$ 0.9 at different values of the drift: $W_{\mathrm{c}}$ decreases with increasing drift and there is also an increase in the residence time dip width. We will analyze the physical reason for the residence time changes with barrier width in detail in Subsecs. III B 2 and III B 3.

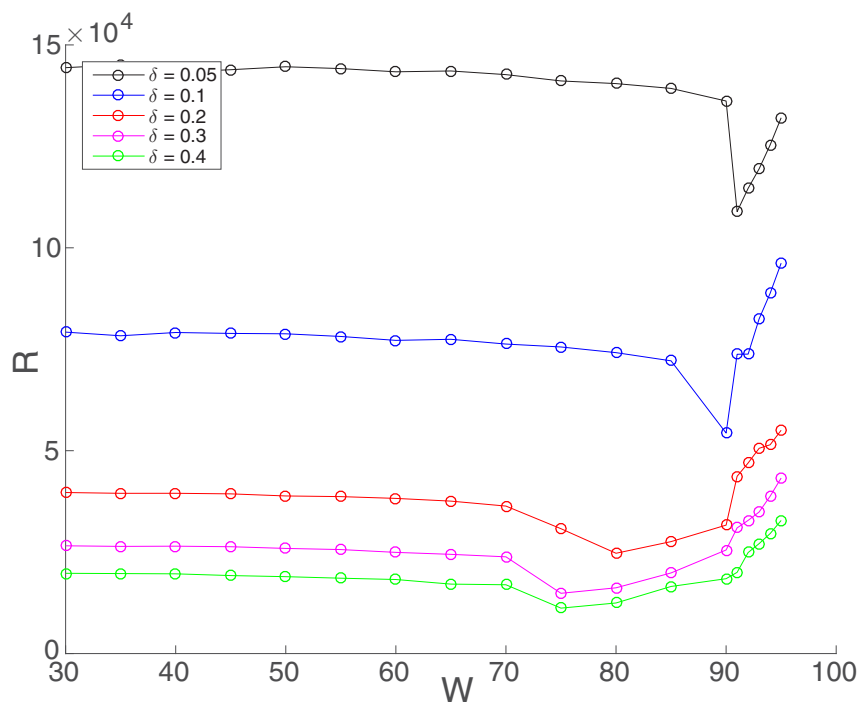

FIG. 9. Simulated mean residence time for the 2D lattice model as a function of the barrier width $W$ for the different values of $\delta$ listed in the inset. Parameters: $L_{1}=100, L_{2}=400, h=0.5, \rho_{\mathrm{u}}=1$, $\rho_{\mathrm{d}}=0.9$, and $O_{2}=3$. 

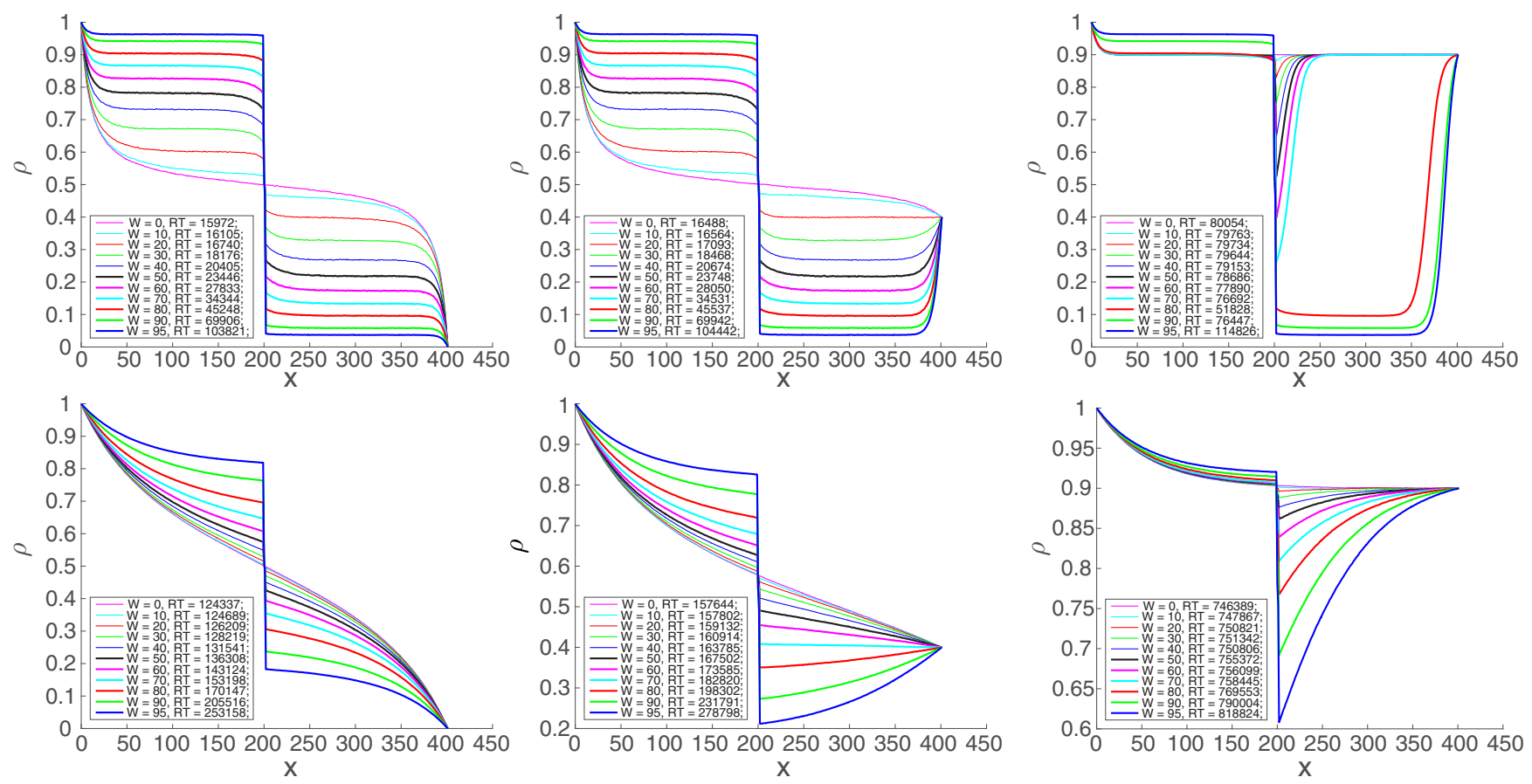

FIG. 10. Density profile obtained by averaging the 2D lattice simulation: comparison for different values of $W$. The lattice size is $100 \times 400, h=0.5, \delta=0.1$ (top) and $\delta=0.01$ (bottom), $\rho_{\mathrm{u}}=1, \rho_{\mathrm{d}}=0$ (left), $\rho_{\mathrm{d}}=0.4$ (center), and $\rho_{\mathrm{d}}=0.9$ (right), and $O_{2}=3$. The Monte Carlo-averaged residence time measured in the different cases has been reported in the inset.

\section{Density profile}

The not uniform dependence of the residence time on $W$ for $\rho_{\mathrm{d}}>0.5$ relates to the asymmetric variation of the density profile before and after the barrier. We will discuss the density profile here and analyze how this relates to the different residence time change regimes in the next section. The differences in density profiles when $W$ is introduced are shown for a few cases in Fig. 10.

In the barrier free case ( $W=0)$, Fig. 10 illustrates the very different density profiles for the nonzero drift case compared to the zero drift case discussed in the previous section. When $\delta=$ 0.1 the slope in density at the center of the strip is substantially decreased compared to the case (see Fig. 4) where diffusion dominates $(\delta=0)$.

When $\delta$ is not zero there is no change in density profiles unless $\rho_{\mathrm{d}} \geqslant 0.5$. As Fig. 10 shows when $\rho_{\mathrm{d}}=0.9$, the density profile in the center becomes equal to $\rho_{\mathrm{d}}$ and has nearly zero derivative. For the decreased value of $\delta=0.01$ the density value at the center is similar, but the slope is larger since diffusion contributes now more to the current. Note (see the date in the inset in Fig. 10) that the ratio between the corresponding values of the residence time is of the same order of the inverse of the ratio between the $\delta$ values.

When a barrier is introduced $(W>0)$, there are important differences between the density changes when $\rho_{\mathrm{d}}$ is smaller or larger than 0.5 . When $\rho_{\mathrm{d}}<0.5$ there is a density increase before the barrier and a wake develops after it (see the left and center panels in Fig. 10). When $\rho_{\mathrm{d}}>0.5$ (see the right panels in Fig. 10), for small $W$ only a wake appears, but at a critical value $W_{\mathrm{c}}$ of the barrier width an increase in density before the barrier is observed.

\section{The physics of the jammed strip with a barrier}

Wake developments after the barrier and density variations before the barrier are sufficient to explain the residence time behavior. In our discussion we will take also advantage of the mean-field approximation for the residence time, which closely follows the trends of the lattice simulated values (see Fig. 11), with an average deviation at high values of $W$ of $1 \%$.

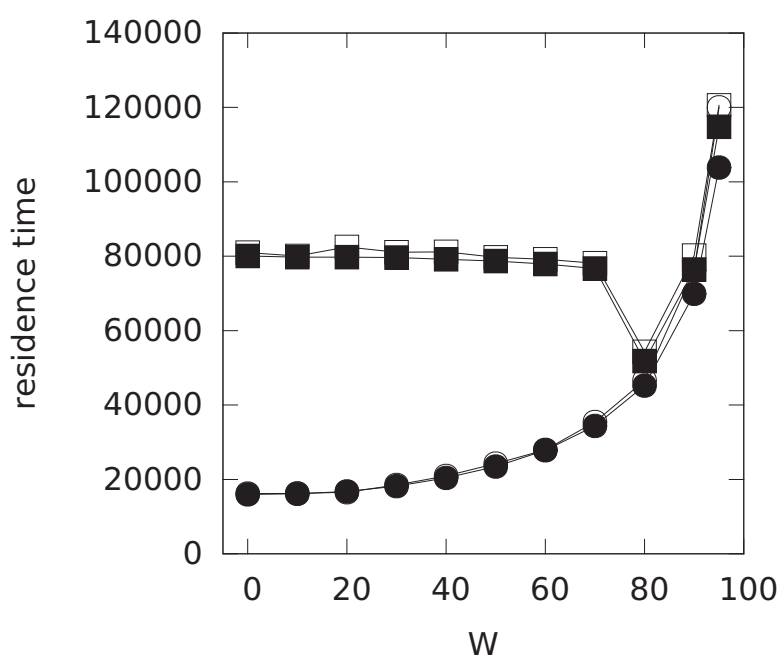

FIG. 11. The Monte Carlo estimate (solid symbols) and the meanfield prediction (open symbols) of the residence time are plotted for $\delta=0.1$, lattice size $100 \times 400, h=0.5, \rho_{\mathrm{u}}=1, O_{2}=3, \rho_{\mathrm{d}}=0$ (circles), and $\rho_{\mathrm{d}}=0.9$ (squares). 


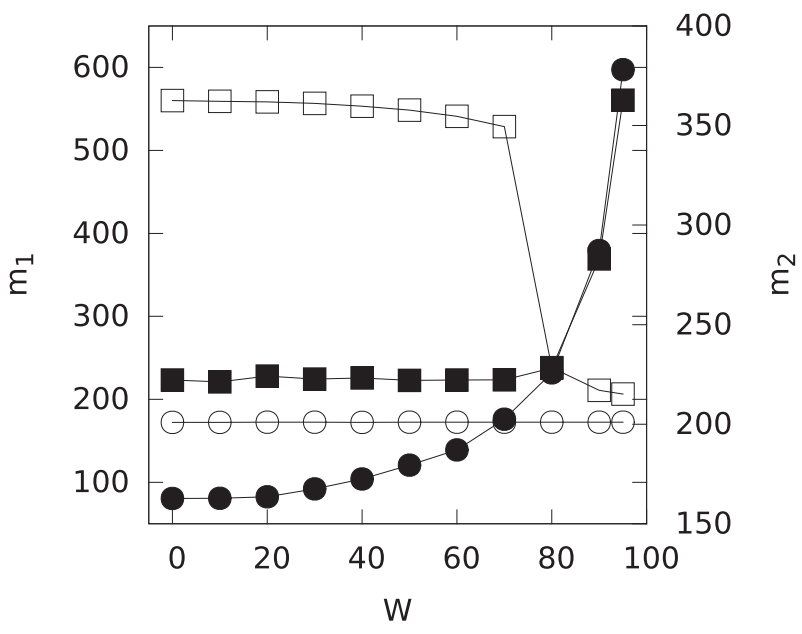

FIG. 12. Plot of the parameters $m_{1}$ (solid symbols) and $m_{2}$ (open symbols) defined in (3.16) for $\delta=0.1$, lattice size $100 \times 400, h=$ $0.5, \rho_{\mathrm{u}}=1, O_{2}=3, \rho_{\mathrm{d}}=0$ (circles), and $\rho_{\mathrm{d}}=0.9$ (squares).

The expression of the residence time based on the meanfield approach can be rewritten as $R=m_{1} m_{2}$ with

$$
m_{1}=-\frac{2}{(1-h) \rho^{\prime}(0)} \text { and } m_{2}=\int_{0}^{L_{2}} \rho(x) d x,
$$

where, we recall, we abuse the notation and denote by $\rho$ the density distribution averaged along the horizontal direction. The quantities $m_{1}$ and $m_{2}$ enable us to distinguish between changes before and after the barrier; indeed, $m_{1}$ changes only when the density profile alters before the barrier, whereas $m_{2}$ measures the total mass in the system.

For $\delta=0.1, \rho_{\mathrm{d}}=0$, and $\rho_{\mathrm{d}}=0.9$ the residence times are plotted in Fig. 11 and the related values of $m_{1}$ and $m_{2}$ are plotted in Fig. 12. The data show the very different dependences of $m_{1}$ and $m_{2}$ as functions of $W$ when $\rho_{\mathrm{d}}=0$ and $\rho_{\mathrm{d}}=0.9$.

Data in Fig. 12 show that, when the outlet density $\rho_{\mathrm{d}}$ is zero, $m_{1}$ increases with $W$ and, hence, the derivative of the density profile at the inlet decreases with $W$. This is connected to the fact that at low outlet density case $\left(\rho_{\mathrm{d}}<0.5\right)$, a linear increase of the density level just before the barrier is observed when the width of the barrier is increased. The onset of percolation before the barrier is thus related to the increase of the parameter $m_{1}$. On the other hand, the parameter $m_{2}$ stays constant, with respect to $W$ for $\rho_{\mathrm{d}}<0.5$. This is shown in the case $\rho_{\mathrm{d}}=0$ in Fig. 12. This fact is due to the symmetric variation of the averaged density profile before and after the barrier around the level 0.5; see the left and center panels in Fig. 10. Hence, the increase in density before the barrier and the decrease after it compensates and $m_{2}$ remains constant. Recalling that $R=m_{1} m_{2}$, we have that in the regime $\rho_{\mathrm{d}}<0.5$ the residence time is an increasing function of $W$.

The response to the introduction of a barrier changes when $\rho_{\mathrm{d}}$ exceeds 0.5. The data plotted in Fig. 12 for $\rho_{\mathrm{d}}=0.9$ show that initially (i.e., when $W$ is small enough) $m_{1}$ is constant and, thus, no change in the derivative of the density at the inlet is observed. This also implies that there is no increase in density before the barrier. For the same values of $W$, on the other hand, the presence of the wake after the barrier decreases the value of $m_{2}$ as $W$ is increased. Thus, this mass loss in the wake after the barrier and the related percolation decrease cause the residence time to decrease as long as the barrier width is less than $W_{\mathrm{c}}$ (see Figs. 9 and 11).

When $W$ increases near the critical value $W_{c}$, the quantity $m_{2}$ steeply decreases as a consequence of the convex to concave transition in the density profile in the wake near the barrier. With a further increase in $W$, the quantity $m_{1}$ starts to increase, which implies that the density starts to increase before the barrier. Residence time now will increase due to increased jamming before the barrier (the decrease of $m_{2}$ is not sufficient to compensate for the increase of $m_{1}$ ).

\section{1D interpretation of the density profiles}

In Subsec. III B 3 we have shown that our numerical results on the residence time can be interpreted using the mean-field approximation and the main features of the stationary density profiles. Here, we show that these profiles can be understood in terms of the 1D model introduced in Sec. II C.

We will discuss the 1D model (2.7)-(2.9) assuming that the lengths $L_{2} \gg O_{2}$ are both large (tend to infinity) so that the transport term dominates diffusion in the expression of the current

$$
J=\lambda\left[-\frac{1}{2} \frac{d \rho}{d x}+\delta \rho(1-\rho)\right] .
$$

Hence, we try to predict the constant bulk values of the density profiles and we describe the small-scale effects close to the boundaries and close to the porosity discontinuity regions. We shall, thus, denote by $\rho_{1}, \rho_{2}$, and $\rho_{3}$ the bulk values of the density profiles in the three regions $x<\left(L_{2}-O_{2}\right) / 2$, $\left(L_{2}-O_{2}\right) / 2<x<\left(L_{2}+O_{2}\right) / 2$, and $x>\left(L_{2}+O_{2}\right) / 2$, respectively. We will discuss how $\rho_{1}, \rho_{2}$, and $\rho_{3}$ depend on the barrier width $W$ under two assumptions: (i) maximization of the bulk current $\lambda \delta \rho(1-\rho)[1,12,18]$ and (ii) $\rho_{1} \geqslant \rho_{\mathrm{d}}$, which is a rather natural assumption in view of the boundary conditions (2.9).

We first remark that (2.8) implies that the current is constant with respect to the space variable; hence, for the bulk values we have that

$$
\rho_{1}\left(1-\rho_{1}\right)=\alpha \rho_{2}\left(1-\rho_{2}\right)=\rho_{3}\left(1-\rho_{3}\right),
$$

where $\alpha \in(0,1]$ has been introduced below Eq. (2.7). Thus we have that

$$
\rho_{1}=\rho_{3} \quad \text { or } \quad \rho_{1}=1-\rho_{3}
$$

and

$$
\rho_{2}=\frac{1}{2}\left[1 \pm \sqrt{1-\frac{4}{\alpha} \rho_{1}\left(1-\rho_{1}\right)}\right],
$$

provided the discriminant is not negative, which is the case if and only if

$$
\rho_{1} \leqslant \frac{1}{2}(1-\sqrt{1-\alpha}) \text { or } \rho_{1} \geqslant \frac{1}{2}(1+\sqrt{1-\alpha}) .
$$

It is important to note that the right-hand side in the second inequality (3.19) varies between 0.5 and 1 for $\alpha \in(0,1]$. The conditions (3.19) have an important physical meaning; indeed, they state that, due to the presence of the reduced porosity in 
the middle of the system, the bulk densities $\rho_{1}$ and $\rho_{3}$ cannot be arbitrarily close to 0.5 .

Consider, now, the case $0 \leqslant \rho_{\mathrm{d}}<0.5$. Since $\rho_{\mathrm{d}}<0.5$ and $(1+\sqrt{1-\alpha}) / 2 \geqslant 0.5$, in order to maximize the current it will be $\rho_{1}=(1+\sqrt{1-\alpha}) / 2$. Thus it will also be $\rho_{2}=$ $1 / 2$ and $\rho_{3}=1-\rho_{1}=(1-\sqrt{1-\alpha}) / 2$. Hence, in this case, independent of the value of $\rho_{\mathrm{d}}$, when $\alpha$ varies from 1 to 0 , that is to say the barrier width varies from 0 to $L_{1}$, the value of $\rho_{1}$ moves from $1 / 2$ to 1 . It is very important to remark that this is precisely what is observed for the horizontally averaged profile of the 2D lattice model in Fig. 10. In particular, note that the bulk density levels are the same in the two top left panels, although they correspond to two different values of the outlet boundary density $\rho_{\mathrm{d}}$.

In the $2 \mathrm{D}$ model the nonlinear changes of residence time that occur upon introduction of the barrier are only observed in the low current regime when $\rho_{\mathrm{d}}>0.5$. As we explained above, this is due to the peculiar behavior of the density profile illustrated in the right top panel in Fig. 10. Now we show that a similar behavior can be conjectured for the bulk density values in the framework of the 1D model.

Thus, consider now the 1D model in the case $\rho_{\mathrm{d}}>0.5$. For $\alpha$ close to 1 one has that $(1+\sqrt{1-\alpha}) / 2<\rho_{\mathrm{d}}$. Hence, the condition $\rho_{1} \geqslant(1+\sqrt{1-\alpha}) / 2$ is not effective and, in order to maximize the bulk current, it will be $\rho_{1}=\rho_{\mathrm{d}}$, which is the smallest value larger than $1 / 2$ that $\rho_{1}$ can assume. Hence, when $\alpha$ is lowered starting from 1 the bulk density $\rho_{1}$ will stay constantly equal to $\rho_{\mathrm{d}}$ until $\alpha$ will be so small that $(1+$ $\sqrt{1-\alpha}) / 2=\rho_{\mathrm{d}}$. This equation defines a critical value of $\alpha$ below which $\rho_{1}=(1+\sqrt{1-\alpha}) / 2$ so that it increases when $\alpha$ is decreased further. In this case, we also have that, since the current must be constant, it will also be $\rho_{3}=1-\rho_{1}=$ $(1-\sqrt{1-\alpha}) / 2$. It is important to note that the behavior here described is precisely the one observed in the right top panel in Fig. 10.

\section{CONCLUSIONS}

We have discussed simulations on a strip lattice model with asymmetric simple exclusion in the presence of a solid impenetrable barrier. We have analyzed these results by averaging the density in a direction perpendicular to the direction of current so that a 1D model could be constructed.

In the 2D model a relative value of the horizontal displacement probability $h=0.5$ has been used. In that case there is rapid horizontal diffusion of density before the barrier to the opening positions between barrier and wall and after barrier into wake region. For the 1D model for zero drift an analytical solution for the residence time could be found in the presence of a barrier. For nonzero drift such an analytical solution could not be found, but the 1D model could be analyzed within the mean-field approximation.

The physics of the increase in residence time by introduction of the barrier for the zero drift case has a simple explanation. We consider the case where the strip inlet particle density is one, but at the outlet of the strip it is varied. In the absence of barrier, when drift is zero, the density between inlet and outlet varies linearly. When a barrier is introduced the density before the barrier increases and a wake develops behind it. Since both changes reduce local density gradients it causes a decrease in current and residence time increases with increase of barrier width $W$. The increase of residence time will occur for any value of $\rho_{\mathrm{d}}$.

The drift contribution to the current depends nonlinearly on the density profile. In the absence of a barrier, when drift is large compared to diffusion, this causes the density to be independent of position except at the inlet and outlet regions of the strip. As long as $\rho_{\mathrm{d}}$ is less than 0.5 the constant bulk value of the density does not change when $\rho_{\mathrm{d}}$ is varied. The current remains maximized since $\rho(1-\rho)$ is maximum for $\rho=0.5$. However, when $\rho_{\mathrm{d}}$ exceeds 0.5 the flat part of the density profile becomes equal to $\rho_{\mathrm{d}}$. The current now decreases and the residence time increases. This is the onset of percolation.

When drift is not zero, the presence of the barrier in the strip gives the expected increase in residence time with increasing barrier width as long as $\rho_{\mathrm{d}}<0.5$. The density before the barrier increases and also a wake develops. The increase in density before the barrier and decrease in density in the wake after the barrier reduce current since densities in both cases start to differ from the value 0.5 that maximizes the bulk current.

However, when $\rho_{\mathrm{d}}>0.5$ the system is already in the percolation regime. Now, the introduction of the barrier can lead to local reduction of the density so that percolation will decrease. The density changes before and after the barrier are now asymmetric. Initially, there is only formation of a wake behind the barrier. This reduction of density reduces percolation and causes an increase of current and a decrease of residence time. There is no increase in density before the barrier since this will increase percolation and hence decreases current. The residence time gets its minimum value when the average value of the density behind the barrier becomes equal to 0.5 ; beyond this point an increase in barrier width will increase the residence time.

Once $W$ increases beyond the critical value $W_{\mathrm{c}}$, where the average density in the wake becomes less than $1-\rho_{\mathrm{d}}$, a further reduction of density in the wake will decrease current similarly as an increase in density before the barrier to higher values of $\rho_{\mathrm{d}}$. Now with increase of barrier width, not only density in the wake decreases but also the density before the barrier will increase. Now, residence time will continue to increase beyond its no-barrier value.

Due to the additional contribution of diffusion, which counteracts the decrease in residence time, the minimum in residence time is reached for larger values of $W$ when the drift is decreased.

In a recent paper [19] the totally asymmetric simple exclusion process has been applied to a molecular motor transport model on a network. Whereas the network is different from our strip model and drift equals one, the authors find also nonlinear dependence on motor particle density when its global density exceeds a critical value and network exit rate is asymmetric. Also, in this case the critical behavior is due to the term $\rho(1-\rho)$ as we discuss in this paper.

\section{ACKNOWLEDGMENTS}

E.N.M.C. acknowledges ICMS (TU/e) for the kind hospitality and financial support. O.K. acknowledges support 
from the Over Grenzen, complexiteit programma, of KNAW (Royal Netherlands Academy of Sciences and Arts). R.v.S. acknowledges support from the NWO Gravitation MCEC programme.
[1] E. N. M. Cirillo, O. Krehel, A. Muntean, R. van Santen, and A. Sengar, Residence time estimates for asymmetric simple exclusion dynamics on strips, Phys. A (Amsterdam, Neth.) 442, 436 (2016).

[2] A. Ahrony and D. Stauffer, Introduction to Percolation Theory (Taylor and Francis, 1994).

[3] J. Sane, J. T. Padding, and A. A. Louis, Taylor dispersion of colloidal particles in narrow channels, Mol. Phys. 113, 2538 (2015).

[4] A. Schadschneider, D. Chowdhury, and K. Nishinari, Stochastic Transport in Complex Systems: From Molecules to Vehicles (Elsevier, 2010).

[5] R. K. P. Zia, E. L. Praestgaard, and O. G. Mouritsen, Getting more from pushing less: Negative specific heat and conductivity in nonequilibrium steady states, Am. J. Phys. 70, 384 (2002).

[6] P. Baerts, U. Basu, C. Maes, and S. Safaverdi, Frenetis origin of negative differential response, Phys. Rev. E 88, 052109 (2013).

[7] D. Braess, Über ein Paradoxon der Verkehrsplanung, Unternehmenforschung 12, 258 (1968).

[8] A. Garcimartin, J. M. Pastor, L. M. Ferrer, J. J. Ramos, C. Martin-Gomez, and I. Zuriguel, Flow and clogging of a sheep herd passing thorugh a bottleneck, Phys. Rev. E 91, 022808 (2015).

[9] I. Zuriguel, D. R. Parisi, R. C. Hidalgo, C. Lozano, A. Janda, P. A. Gago, J. P. Peralta. L. M. Ferrer, L. A. Pugnaloni, E. Clement, D. Maza, I. Pagonabarraga, and A. Garcimartin, Clogging transition of many-particle systems flowing through bottlenecks, Sci. Rep. 4, 7324 (2014).
[10] D. Helbing, L. Buzna, A. Johansson, and T. Werner, Selforganized pedestrian crowd dynamics: Experiments, simulations, and design solutions, Trans. Sci. 39, 1 (2005).

[11] K. Suzuno, A. Tomoeda, M. Iwamoto, and D. Ueyama, Dynamic structure in pedestrian evacuation: Image processing approach, Traffic and Granular Flow '13, edited by M. Chraibi, M. Boltes, A. Schadschneider, and A. Seyfrid (Springer International Publishing, Switzerland, 2015), pp. 195-201.

[12] J. Krug, Boundary-Induced Phase Transition in Driven Diffusive Systems, Phys. Rev. Lett. 67, 1882 (1991).

[13] A. B. Kolomeisky, Asymmetric simple exclusion model with local inhomogeneity, J. Phys. A 31, 1153 (1998).

[14] P. Greulich and A. Schadschneider, Phase diagram and edge effects in the ASEP with bottlenecks, Phys. A (Amsterdam, Neth.) 387, 1972 (2008).

[15] M. J. Simson, K. A. Landman, and B. D. Hughes, Multispecies simple exclusion processes, Phys. A (Amsterdam, Neth.) 388, 399 (2015).

[16] P. Bastian, M. Blatt, A. Dedner, C. Engwer, R. Klöfkorn, R. Kornhuber, M. Ohlberger, and O. Sander, A generic grid interface for parallel and adaptive scientific computing, part II: Implementation and tests in DUNE, Computing 82, 121 (2008).

[17] J. Bear, Dynamics of Fluids in Porous Media (Dover, 1972).

[18] M. R. Evans, N. Rajewsky, and E. R. Speer, Exact solution of a cellular automaton for traffic, J. Stat. Phys. 95, 45 (1999).

[19] D. V. Denisov, D. M. Miedema, B. Nienhuis, and P. Schall, Phys. Rev. E 92, 052714 (2015). 